\title{
Nanocomposite hydrogel-based biopolymer modified with silver nanoparticles as an antibacterial material for wound treatment
}

\author{
Helmiyati*, Gissi Novientri, Gusma Harfiana Abbas, Emil Budianto \\ Department of Chemistry, Faculty of Mathematics and Natural Sciences, Universitas Indonesia, Depok 16424, West Java, Indonesia.
}

\section{ARTICLE INFO \\ Received on: 04/08/2019 \\ Accepted on: 18/09/2019 \\ Available online: 04/11/2019}

Key words:

AgNPs, antibacterial,

hydrogel, nanocomposite, sodium alginate.

\begin{abstract}
Authors report the synthesis of sodium alginate-polyvinyl alcohol-g-acrylamide (NaAlg-PVA-g-AAm) nanocomposite hydrogels modified with silver nanoparticles (AgNPs) as an antibacterial agent. In this work, we used NaAlg isolated directly from brown algae and studied the effects of the NaAlg weight ratio and silver-ion concentration on the network matrix in the hydrogels via in situ polymerization. Successfully synthesized nanocomposites were characterized using Fourier transform infrared, scanning electron microscopy, transmission electron microscopy, X-ray diffraction, and atomic absorption spectrometry. The best results were achieved with an average AgNPs size of approximately $20 \mathrm{~nm}$ allowing the AgNPs to be absorbed in the nanocomposite hydrogel matrix. Nanocomposite hydrogels displayed good antibacterial activity against Escherichia coli and Staphylococcus aureus. The minimum inhibitory concentrations (MICs) of silver nitrate $\left(\mathrm{AgNO}_{3}\right)$ for $E$. coli and $S$. aureus were 46.251 and $75.220 \mathrm{ppm}$, respectively. Conversely, the minimum bactericidal concentrations (MBCs) of $\mathrm{AgNO}_{3}$ for these bacteria were 185.004 and $300.880 \mathrm{ppm}$, respectively. The $\mathrm{MBC} / \mathrm{MIC}$ ratio of the $\mathrm{AgNO}_{3}$ modified nanocomposite hydrogels was four for both bacteria. The results illustrated that the nanocomposite hydrogels had good antibacterial activity against Gram-positive and Gramnegative bacteria and can be suitable for applications in wound treatments.
\end{abstract}

\section{INTRODUCTION}

Many recent studies have shown the benefits of using hydrogels for wound care, as they can maintain a moist environment around the wound site and improve the healing process by protecting damaged skin from dehydration (GhavamiNejad et al., 2016). Ideal wound treatment systems are based on the principle of moist wound healing, eliminating the excess fluid in the wound such that the balance of moisture can be maintained. This can be achieved by using hydrogels (Jayakumar et al., 2011). Hydrogels are structurally similar to the extracellular matrix of living tissue and therefore have extensive applications in wound healing (Lee and Mooney, 2012). The use of hydrophilic polymers such as sodium alginate $(\mathrm{NaAlg})$ as raw materials for hydrogel is interesting because this compound is non-toxic, is abundant

"Corresponding Author

Helmiyati Manjas, Department of Chemistry, Faculty of Mathematics and Natural Sciences, Universitas Indonesia, Depok 16424, West Java, Indonesia.E-mail: helmi-yt@ui.ac.id in nature, and is derived from seaweed. NaAlg can therefore, be used to develop hydrogels that are renewable, biodegradable, biocompatible, and not immunogenic (Gharekhania et al., 2017; Raguvaran et al., 2017; Thakur et al., 2018). The unique properties of sodium alginate and its relatively low cost have made it one of the most interesting materials in pharmaceutical, biomedical, and agricultural applications (Gharekhania et al., 2017; Pereira et al., 2013). Polyvinyl alcohol (PVA) is a synthetic polymer that is often used with natural polymers to improve the mechanical performance of natural polymers. PVA has several advantages: it is non-toxic, has good mechanical properties, and is easy to process (Frone et al., 2015). Given the nature of these two polymers, it is expected that a combination of NaAlg and PVA can yield a good hydrogel structure that can be used as an effective nanoreactor (Ghasemzadeh and Ghanaat, 2014). Hydrogel polymers are hydrophilic compounds with three-dimensional tissue structures that are able to absorb, expand, and can retain large amounts of liquid (Fekete et al., 2017; Helmiyati et al., 2014). Hydrogels contain hydrophilic polymer groups that can be used to synthesize nanoparticles because they have empty spaces/pores between their crosslinked tissues, which make them act as nanoreactors (El-Sherif et al., 2011). 
The use of antibacterial agents such as nano-sized metals and metal oxides can prevent the growth of microorganisms (Zhao et al., 2018). Silver nanoparticles (AgNPs) have emerged as important nanomaterials for a variety of industrial and medical applications but they also present potential risks to human health (Ahamed et al., 2010). Therefore, a hydrogel can be used as a controlled release system by functioning as a matrix for AgNP nanoreactors, which would allow for controlled release of AgNPs or $\mathrm{Ag}^{+}$ions, thus limiting the potential risks of excess release of nanoparticles (Basu et al., 2017). Antibacterial activity is an important characteristic of wound treatment materials because the fluid in the wound is a good medium for bacterial growth. Thus, using the hydrogel matrix as a nanoreactor can lead to a more controlled release of $\mathrm{AgNPs}_{\mathrm{Ag}} \mathrm{Ag}^{+}$ions (Veiga et al., 2013).

In this study, the nanocomposite hydrogel NaAlg-PVA was synthesized using NaAlg isolated from brown algae in Banten, Indonesia, which we reported previously (Helmiyati et al., 2017). The NaAlg was combined with PVA and then grafted with an acrylamide monomer (AAm) and N, N'-methylenbisacrilamide (MBA) as crosslinking agents, and ammonium persulfate as an initiator. The effect of the ratio of sodium alginate to PVA was studied and the hydrogel was modified with AgNPs to obtain properties. This work analyzed the $\mathrm{Ag}^{+}$ion release capacity in water by varying the loading

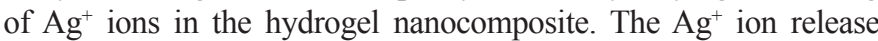
capacity is important because it relates to the ability of the hydrogel matrix to release $\mathrm{Ag}^{+}$ions in a controlled manner to inhibit bacterial growth. The swelling capacity of the AgNP-modified hydrogel nanocomposite was also studied, and antibacterial activity against Staphylococcus aureus (Gram-positive bacteria) and Escherichia coli (Gram-negative bacteria) was measured in vitro.

\section{MATERIALS AND METHODS}

\section{Materials}

$\mathrm{NaAlg}$ from brown algae with a molecular weight of 40,680 g/mol (Helmiyati et al., 2017), polyvinyl alcohol (PVA) with a deacetylation degree of around $86.5 \%$ and molecular weight about $180 \mathrm{kDa}$ (Lubon Vinylon, Co). The bacterial growth medium consisted of nutrient agar for microbiology (Merck \& Co.), ISO 6579 peptone water (Merck \& Co.), and Lab-Lemco beef extract powder (Oxoid Ltd.). Chloramphenicol (Bernofarm Pharmaceutical) was used as a positive control. $S$. aureus was cultured by the School of Pharmacy of ITB in Bandung, Indonesia, and E. coli was cultured by the Faculty of Medicine of Indonesia University. All other chemical reagents were purchased from Sigma-Aldrich.

\section{Synthesis of the NaAlg-PVA-g-AAm hydrogel and modification with AgNPs}

The NaAlg-PVA-g-AAm hydrogel was synthesized as previously described (Ghasemzadeh and Ghanaat, 2014), with slight modifications. The NaAlg solution was added to the reactor (three-neck flask) using a magnetic stirrer, reflux condenser, thermometer, and nitrogen gas. Potassium persulfate or KPS $(5 \mathrm{ml}, 0.75 \% \mathrm{w} / \mathrm{v})$ was added to the reactor and stirred for 5 minutes, after which the mixture was dried under streaming nitrogen gas for 5 minutes. Then, PVA (1 g), AAm (6 ml, 20\% $\mathrm{w} / \mathrm{v})$, and MBA $(10 \mathrm{ml}, 0.75 \% \mathrm{w} / \mathrm{v})$ were added to the reaction mixture. The reaction was allowed to stand until polymerization completed. The product was dried in the oven at $50^{\circ} \mathrm{C}$ until a constant weight was achieved. The process was carried out with various ratios of sodium alginate to PVA: Hyd 1 (1:1), Hyd 2 (2:1), and Hyd 3 (3:1). Hyd 3 yielded the best hydrogel. After achieving an optimal sodium alginate/PVA ratio, the hydrogel nanocomposite was modified with various concentrations of silver nitrate.

Modification of the nanocomposite hydrogel with AgNPs was performed as previously described (Deen et al., 2015; Ghasemzadeh and Ghanaat, 2014). Dried hydrogel was submerged in $\mathrm{AgNO}_{3}$ solution for 24 hours, followed by the addition of $10 \mathrm{mM} \mathrm{NaBH}_{4}$ solution and incubation for 3 hours at room temperature to reduce the silver ions to nanoparticles. The formation of the AgNP-modified hydrogel was indicated by a change in the color of the hydrogel from white to light brown. The variations in the composition of the AgNP-modified hydrogel (HNC.3) were performed by varying $\mathrm{AgNO}_{3}$ concentration from 6.25 to 1,000 ppm (HNC.3a-HNC.3g).

\section{Swelling capacity in water}

The swelling capacity was determined as previously described (Helmiyati et al., 2014). Dried hydrogel (0.1 g) was inserted into nylon fabric and submerged in $200 \mathrm{ml}$ of distilled water at room temperature. The swelling capacity was calculated with Equation (1) as follows:

$$
S(\%)=\frac{m_{s t}-m_{0}}{m_{0}} \times 100 \%
$$

where $S$ is the swelling capacity, $\mathrm{m}_{\mathrm{o}}$ is the initial mass of the dried hydrogel, and $\mathrm{m}_{\mathrm{st}}$ is the hydrogel mass after swelling at certain times.

\section{$\mathrm{Ag}^{+}$ion release capacity}

The $\mathrm{Ag}^{+}$ion release capacity was determined using the atomic absorption spectrometry (AAS) AA-6300 Shimadzu device with a hollow $\operatorname{Ag}(\mathrm{I})$ cathode lamp at a wavelength of $328.07 \mathrm{~nm}$ and an external standard series. The release capacity was measured by dipping the nanocomposite hydrogels in water as a model. The release capacity was calculated with Equation (2) as follows:

$$
\mathrm{CR}\left(\frac{\mathrm{ppm}}{g}\right)=\frac{\mathrm{CR}(\mathrm{ppm})}{m}
$$

where $\mathrm{CR}$ is the $\mathrm{Ag}^{+}$ion release concentration and $\mathrm{m}$ is the hydrogel mass used.

\section{$\mathrm{Ag}^{+}$ion loading}

The $\mathrm{Ag}^{+}$ion loading was determined using an AAS instrument similar to the release capacity. $\mathrm{Ag}^{+}$ion loading in the hydrogel was calculated with Equation (3) as follows:

$$
\text { Loading }\left(\frac{\mathrm{ppm}}{g}\right)=\frac{\left(C_{0}-C_{\mathrm{s}}\right)}{m}
$$

where $C_{\mathrm{o}}$ is the initial concentration of $\mathrm{AgNO}_{3}, C_{\mathrm{s}}$ is the remaining concentration of silver nitrate solution after loading $\mathrm{Ag}^{+}$ions, and $m$ is the hydrogel mass used.

\section{Antibacterial activity assay}

An antibacterial activity assay was performed with the nanocomposite hydrogel modified with AgNPs using different 
concentrations of $\mathrm{AgNO}_{3}$. The medium used to grow the bacterial suspension was a nutrient broth containing ISO 6579 peptone water and Lab-Lemco beef extract powder. Nutrient agar was used as a solid medium to regenerate bacterial growth. $S$. aureus and $E$. coli were used as representative Gram-positive and Gramnegative bacteria, respectively. Encapsulated chloramphenicol was used as a positive control and the negative control was the absence of AgNP-modified hydrogel nanocomposite. The sample was incubated for 24 hours at $37^{\circ} \mathrm{C}$, after which the optical density (OD) of the samples was measured at $625 \mathrm{~nm}$ using a UV/VIS LLG Spectrophotometer (uniSPEC 4). The OD can be used to calculate the percent inhibition and bacterial cell death based on equations 4 and 5, respectively:

$$
\begin{aligned}
& \text { Inhibition }(\%)=\frac{\text { ODN }- \text { ODS }}{\text { ODN }} \times 100 \% \\
& \text { Bacterial death }(\%)=\frac{\text { ODN }- \text { ODS }}{\text { ODN }- \text { ODP }} \times 100 \%
\end{aligned}
$$

where ODN is the optical density of the negative control, ODP is the optical density of the positive control, and ODS is the optical density of the nanocomposite.

\section{RESULTS AND DISCUSSION}

\section{Synthesis of the NaAlg-PVA-g-AAm nanocomposite hydrogel modified with AgNPs}

In this study, the NaAlg-PVA-g-AAm nanocomposite hydrogel was synthesized by grafting PVA and AAm on the NaAlg backbone with the cross-linking agent MBA. The mechanism of grafting with free radical polymerization generally involves the formation of a free-radical active center in the backbone polymer. In this case, the backbone polymer is sodium alginate. The formation of alginate macroradical polymers was initiated using KPS and continued with propagation and termination (Ghasemzadeh and Ghanaat, 2014; Helmiyati et al., 2010).

The proposed mechanism of polymerization of the NaAlg-PVA-g-AAm nanocomposite hydrogel is shown in Figure 1.

The polymerization process (Fig. 1a) was initiated with KPS and heating, resulting in sulfate radical formation. Afterward, the sulfate radicals were attacked on alginate, which initiated the formation of alginate macroradicals. During the propagation phase (b), the polymer chain was elongated. The activation of alginate macroradicals and their reaction with AAm and PVA result in the formation of alginate-AAm-PVA complex radicals. Next, in

a)
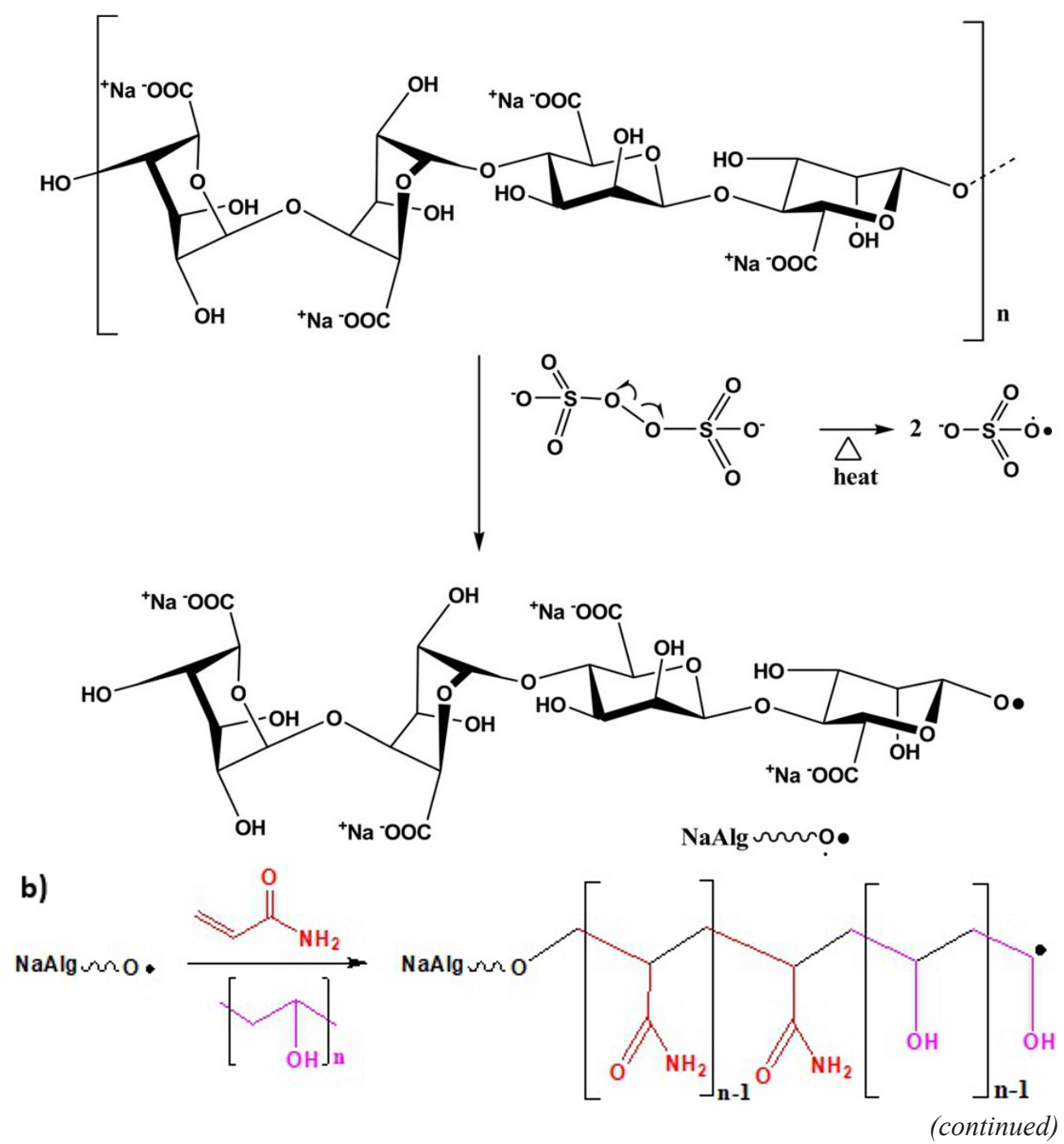
c)

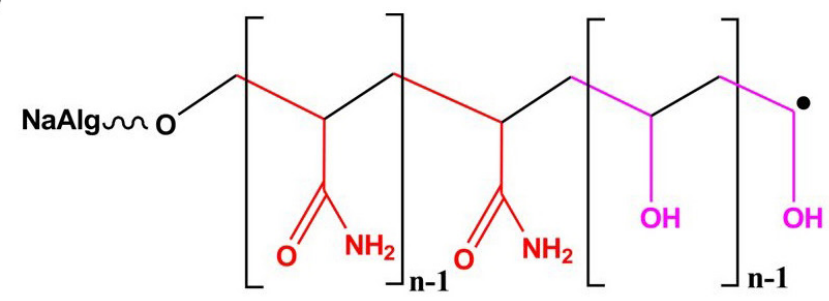

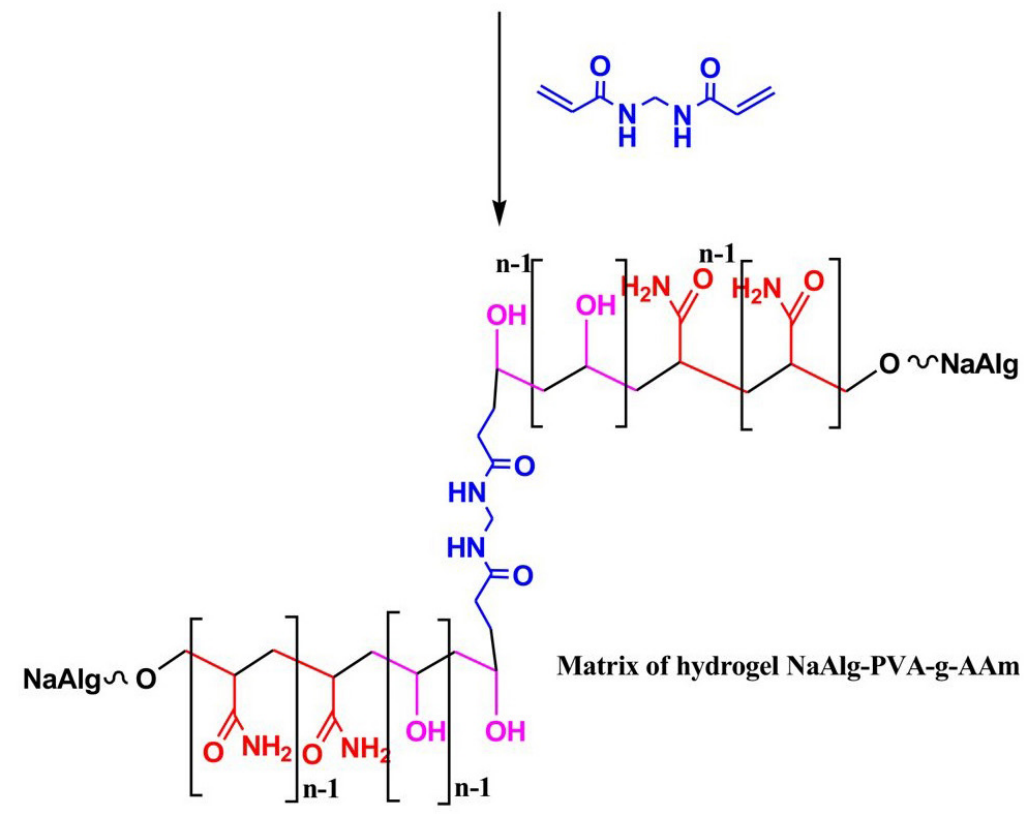

Figure 1. Polymerization stages. (a) Initiation, (b) Propagation, and (c) Termination.

(a)

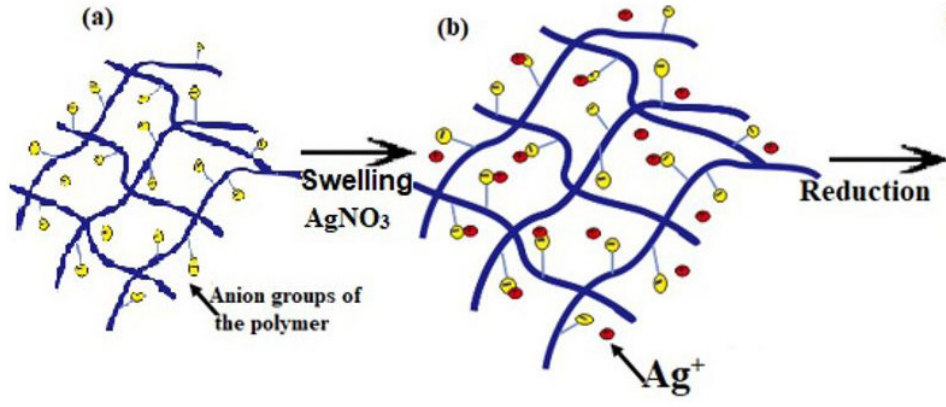

(c)

)

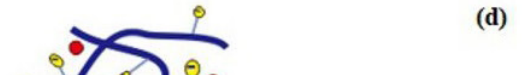

(d)

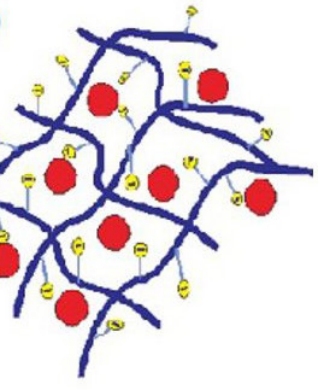

Figure 2. Illustration of NaAlg-PVA-g-AAm nanocomposite hydrogel modified with AgNPs.

the termination stage (c), the addition of the cross-linking agent MBA terminated the elongation of the polymer chain. MBA can combine complex radicals to form three-dimensional networks known as hydrogels. The process of grafting and cross-linking causes an increase in solution viscosity (Zhao et al., 2017).

The synthesized nanocomposite hydrogel has a network that can be used as a template for the formation of AgNPs. An illustration of hydrogel modification with AgNPs is shown in Figure 2.

In the first stage, the hydrogel matrix was immersed in a solution of $\mathrm{AgNO}_{3}$ nanoparticle precursors (a). During the second stage, diffusion of $\mathrm{Ag}^{+}$ions into the hydrogel network caused swelling of the hydrogel matrix because of the difference in concentration between the precursor solution and the interior of the hydrogel network (b). The insertion of $\mathrm{Ag}^{+}$ions was also caused by electrostatic interactions between the $\mathrm{Ag}^{+}$ions and the carboxylate anions $\left(\mathrm{COO}^{-}\right)$inside the hydrogel network. In the third stage (c), $\mathrm{Ag}^{+}$ions in the swollen hydrogel were reduced to $\mathrm{Ag}^{0}$ with the reduction agent $\mathrm{NaBH}_{4}$. The reduction of $\mathrm{Ag}^{+}$ions to $\mathrm{Ag}^{0}$ can be described by Equation (6) (Solomon et al., 2007).

$$
\mathrm{AgNO}_{3}+\mathrm{NaBH}_{4} \rightarrow \mathrm{Ag}^{0}+\frac{1}{2} \mathrm{H}_{2}+\frac{1}{2} \mathrm{~B}_{2} \mathrm{H}_{6}+\mathrm{NaNO}_{3}
$$

In the last stage (d) in Figure 2, the $\mathrm{Ag}^{0}$ formed an aggregate leading to atomic cluster-cluster formation because of the metal bonds. The integration of atoms or nucleation stops 


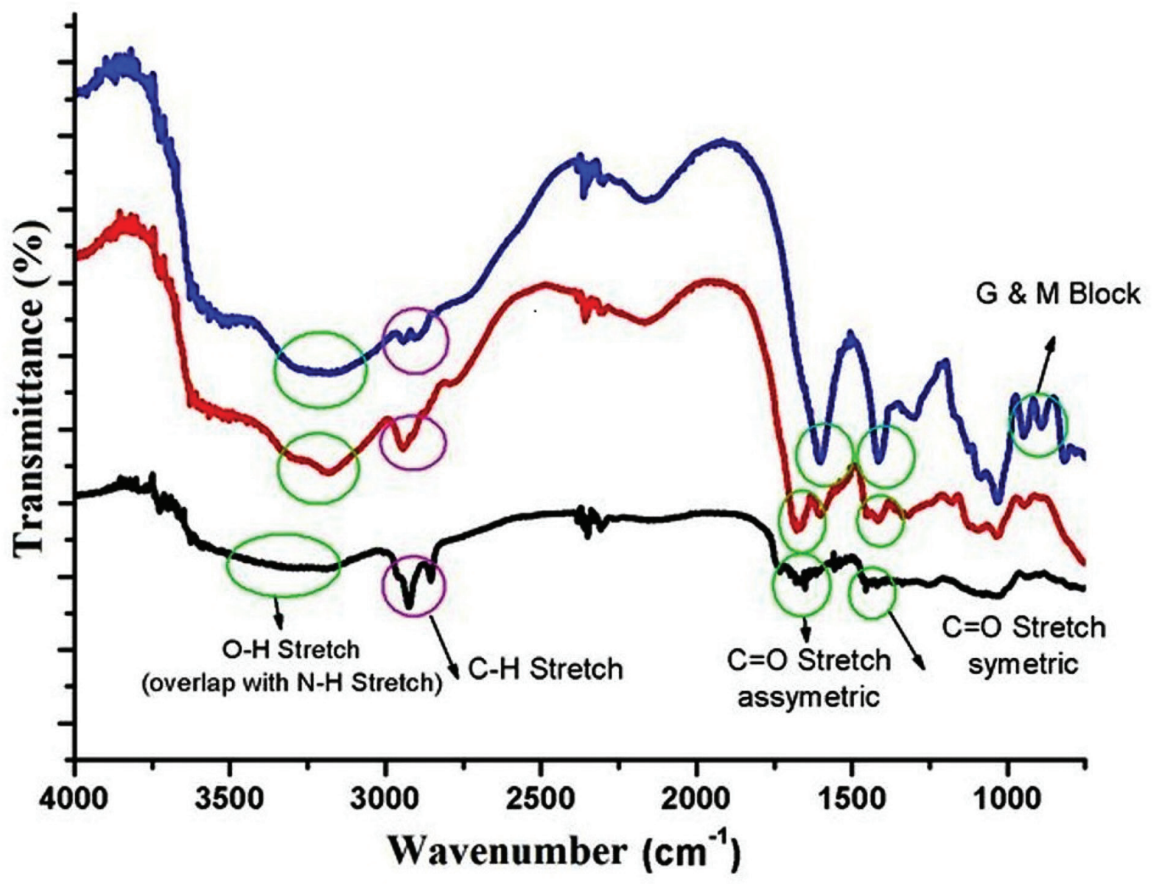

Figure 3. FTIR spectrum of NaAlg (blue); Hyd. NaAlg-PVA-g-gAAM (red); Hyd. modified with AgNPs (black).

depending on the number and size of pores inside the hydrogel network. The hydrogel can serve as a template and stabilizer for the nanoparticles because of the polymer chains between insulated pores likely act as dividers that can minimize the aggregation of Ag particles so that the nanoparticles remain small.

\section{FTIR analysis}

Analyses of the IR spectrum of sodium alginate from brown algae (NaAlg), the NaAlg-PVA-g-AAm hydrogel without modification (Hyd. 3), and the nanocomposite hydrogel modified with AgNPs (HNC.3) are presented in Figure 3.

The Fourier transform infrared (FTIR) spectrum of NaAlg (blue) showed a broad peak at $3,000-3,400 \mathrm{~cm}^{-1}$ that is specific for the stretch vibration of a hydroxyl group $(\mathrm{O}-\mathrm{H})$. The peaks at 1,579 and $1,400 \mathrm{~cm}^{-1}$ reflect asymmetric and symmetric stretch from carbonyl $(\mathrm{C}=\mathrm{O})$ groups on carboxylate anions $\left(\mathrm{COO}^{-}\right)$. The NaAlg structure was divided into two types, $\mathrm{M}$ and $\mathrm{G}$. The $\mathrm{M}$ type consisted of D-mannuronic acid, while the G type consisted of $\mathrm{L}$-guluronic acid. The proportion of $\mathrm{M}$ and $\mathrm{G}$ subtypes of NaAlg structure was identified from the IR absorption band characteristics. Peaks at 828 and $960 \mathrm{~cm}^{-1}$ were observed for $\mathrm{C}-\mathrm{H}$ mannuronic acid and C-H guluronic acid, respectively (Helmiyati et al., 2017). The FTIR spectrum of Hyd.NaAlg-PVA-g-gAAM (Hyd. 3) (red) reflects hydrophilic groups such as $\mathrm{O}-\mathrm{H}$ at $3,000-3,400 \mathrm{~cm}^{-1}$ and carbonyl groups $(\mathrm{C}=\mathrm{O})$ at 1,600 and $1,400 \mathrm{~cm}^{-1}$ from the NaAlg backbone; these peaks are slightly displaced due to the interaction between NaAlg and another monomer in the hydrogel. The presence of PVA polymer grafting on NaAlg is reflected by a stretch on the $\mathrm{O}-\mathrm{H}$ group, which was increased in intensity, indicating that the amount of $\mathrm{O}-\mathrm{H}$ in the structure was higher because of the addition of the O-H group from PVA. The intensity in the $3,000-3,500 \mathrm{~cm}^{-1}$ range was also caused by overlapping with the peak for the amine group $\left(-\mathrm{NH}_{2}\right)$ from the acrylamide monomer. Interactions among the monomers inside the hydrogel are reflected by the larger peak of the $\mathrm{C}-\mathrm{H} \mathrm{sp} 3$ bond at 2,900 $\mathrm{cm}^{-1}$. The spectrum for the AgNPmodified hydrogel (black) reflects the insertion of $\mathrm{Ag}^{+}$ions into the NaAlg-PVA-g-AAm hydrogel network, which causes physical interactions between the $\mathrm{Ag}^{+}$ions and the hydrophilic group inside the pores of the hydrogel network. Peaks for hydrophilic groups, such as $\mathrm{O}-\mathrm{H}, \mathrm{N}-\mathrm{H}$, and carboxylate group $(\mathrm{C}=\mathrm{O})$ stretches exhibited decreased intensity compared with the spectra of the unmodified NaAlg-PVA-g-AAm hydrogel. FTIR characterization results can provide evidence for the successful formation of $\mathrm{Ag}$ nanoparticles within the hydrogel network.

\section{SEM analysis}

Scanning electron microscopy (SEM) was used to characterize the three-dimensional surface morphology of the nanocomposite hydrogel, revealing a porous structure. The presence of pores is closely related to the ability of the hydrogel to swell and absorb water. The hydrogel is able to absorb water because the pores are not only present on the surface but also inside the polymer body. Spaces with pores allow for the absorption of water on the surface; the water then diffuses into the center of the gel due to the capillary force (Zhao et al., 2014). The SEM characterization is presented in Figure 4.

Hydrogels with Alg/PVA ratios of 1:1 (Hyd. 1), 2:1 (Hyd. 2), and 3:1 (Hyd. 3) are presented in Figure 4a-c. It can be seen that the composition of sodium alginate used in the synthesis of hydrogels affects the production of pores, with higher concentrations of alginate resulting in more uniform pores (Fig. 4c). A higher concentration of alginate leads to 

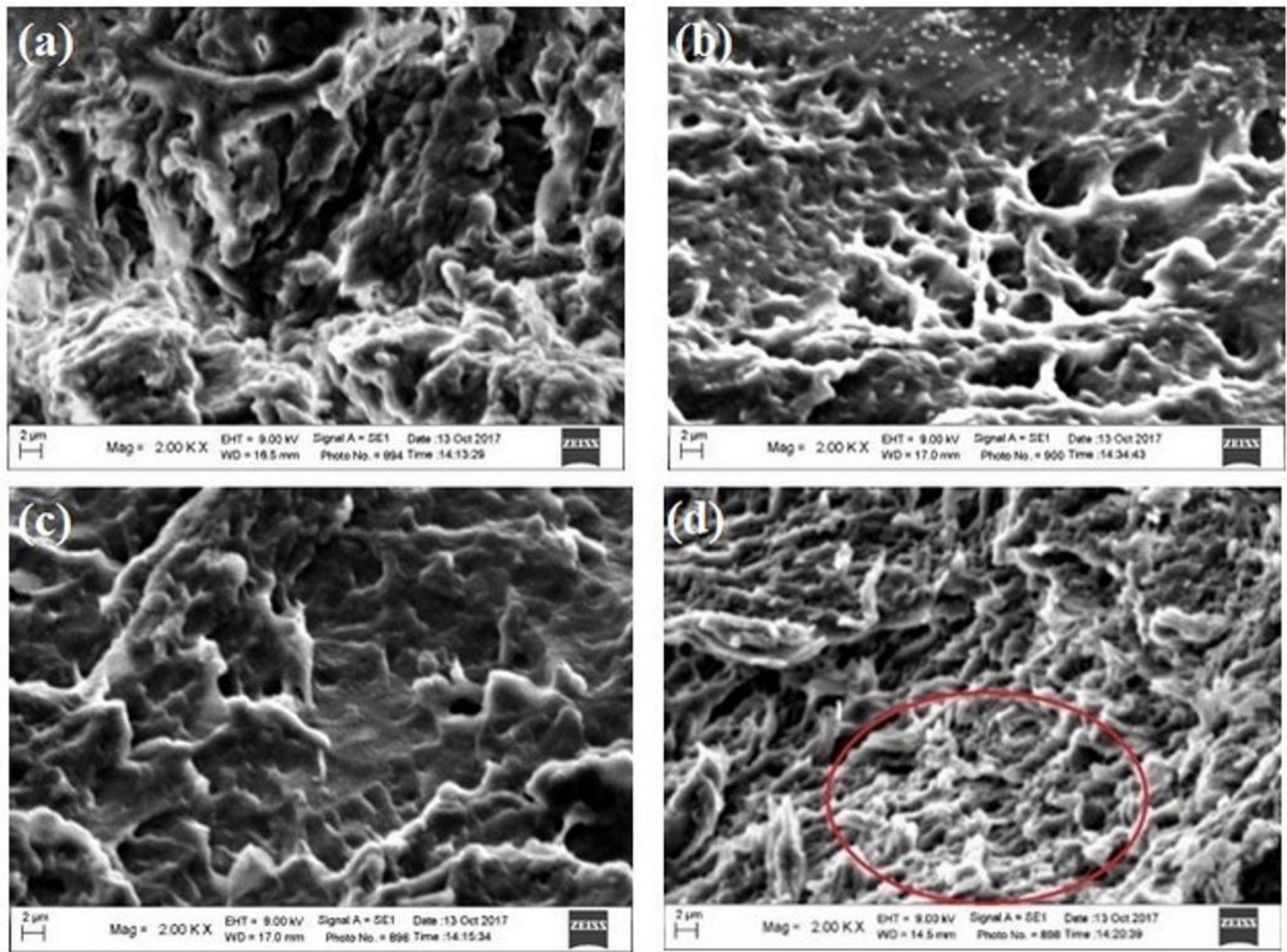

Figure 4. Morphological analysis by SEM. (a) Hyd. 1, (b) Hyd. 2, (c) Hyd. 3, and (d) HNC (red cycle: pores are more tightly filled with AgNP at $200 \times$ Magnification).

a higher number of alginate radicals, which in turn yields more numerous and more uniform pores. Higher Alg/PVA ratios affected the number of pores produced during hydrogel synthesis (Fekete et al., 2017; Ghasemzadeh and Ghanaat, 2014). Figure 4d shows nanocomposite hydrogels modified with AgNPs and demonstrates the formation of silver nanoparticles in the hydrogels. The AgNPs can fill the pores in the hydrogel so that they appear to be tighter.

\section{TEM analysis}

TEM analysis was performed to characterize the twodimensional surface morphology and determine particle shape and size (Fig. 5).

Figure 5a (scale $50 \mathrm{~nm}$ ) shows standard AgNPs with spherical particles that are uniform in size and the average particle size was approximately $20 \mathrm{~nm}$. The AgNPs inside the nanocomposite hydrogel matrix were nearly spherical. As shown in Figure $5 \mathrm{~b}$ (scale $100 \mathrm{~nm}$ ), the average particle size of the AgNPs in the hydrogel matrix was approximately $20 \mathrm{~nm}$.

The SEM and TEM results confirmed that the hydrogel pore structure could be used as a nanoreactor to synthesize AgNPs because the size of the nanoparticles produced depends on the size of the pores inside the hydrogel.

\section{XRD analysis}

The X-ray diffraction (XRD) diffraction pattern of the hydrogel modified with AgNPs (HNC) presented in Figure 6 showed four peaks at $37.46^{\circ}, 45.94^{\circ}, 64.45^{\circ}$, and $72.64^{\circ}$ reflecting the AgNPs, the peak for the AgNP-modified hydrogel is similar to that for standard AgNPs (black) and peaks at $2 \theta 13.58^{\circ}$ and $22.05^{\circ}$ reflecting NaAlg (Helmiyati et al., 2017). The peaks were shifted due to the addition of AgNPs inside the hydrogel matrix. Thus, it can be seen that AgNPs were formed in the hydrogel matrix. However, the diffraction pattern of the AgNPs modified nanocomposite hydrogel also shows a peak at $32.10^{\circ}$ (circled in purple), it is possible that $\mathrm{Ag}_{2} \mathrm{O}$ nanoparticles form when the nanocomposite hydrogels are drying (Phongtongpasuk et al., 2016). The size of AgNPs in the nanocomposite hydrogels as determined using the DebyeScherrer equation was approximately $20 \mathrm{~nm}$, consistent with the results of the TEM analysis. 

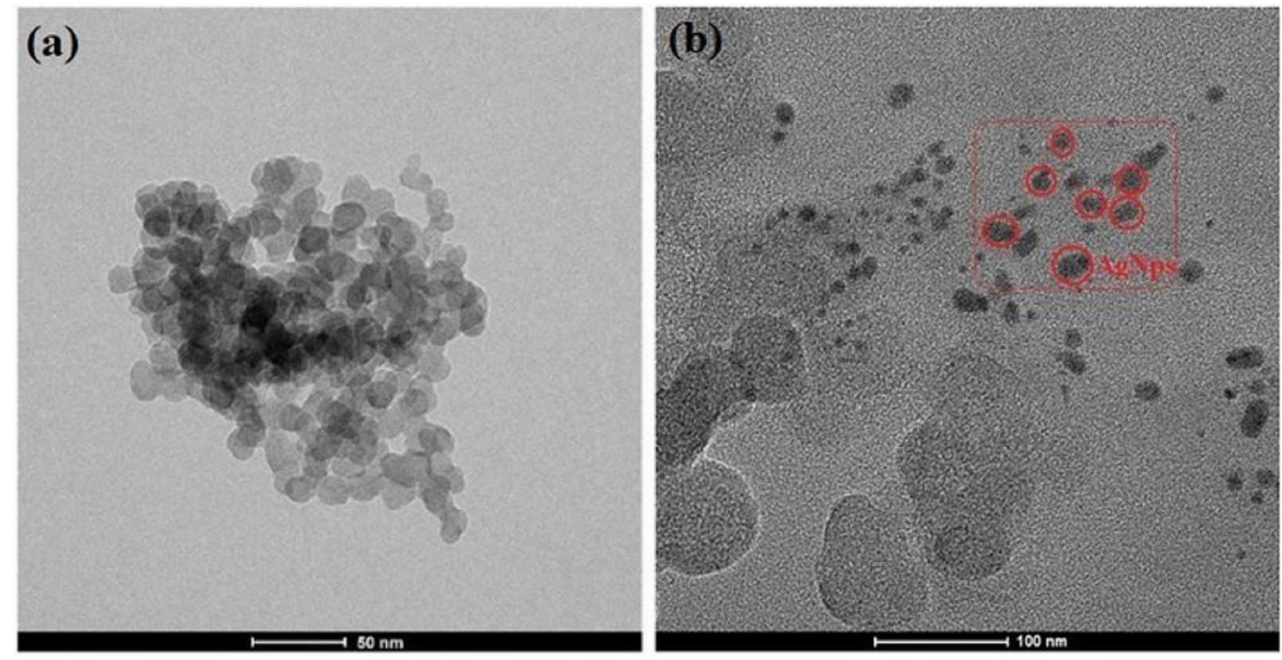

Figure 5. TEM results for (a) standard AgNps, $50 \mathrm{~nm}$ scale and (b) AgNPs in a hydrogel matrix (HNC), $100 \mathrm{~nm}$ scale.

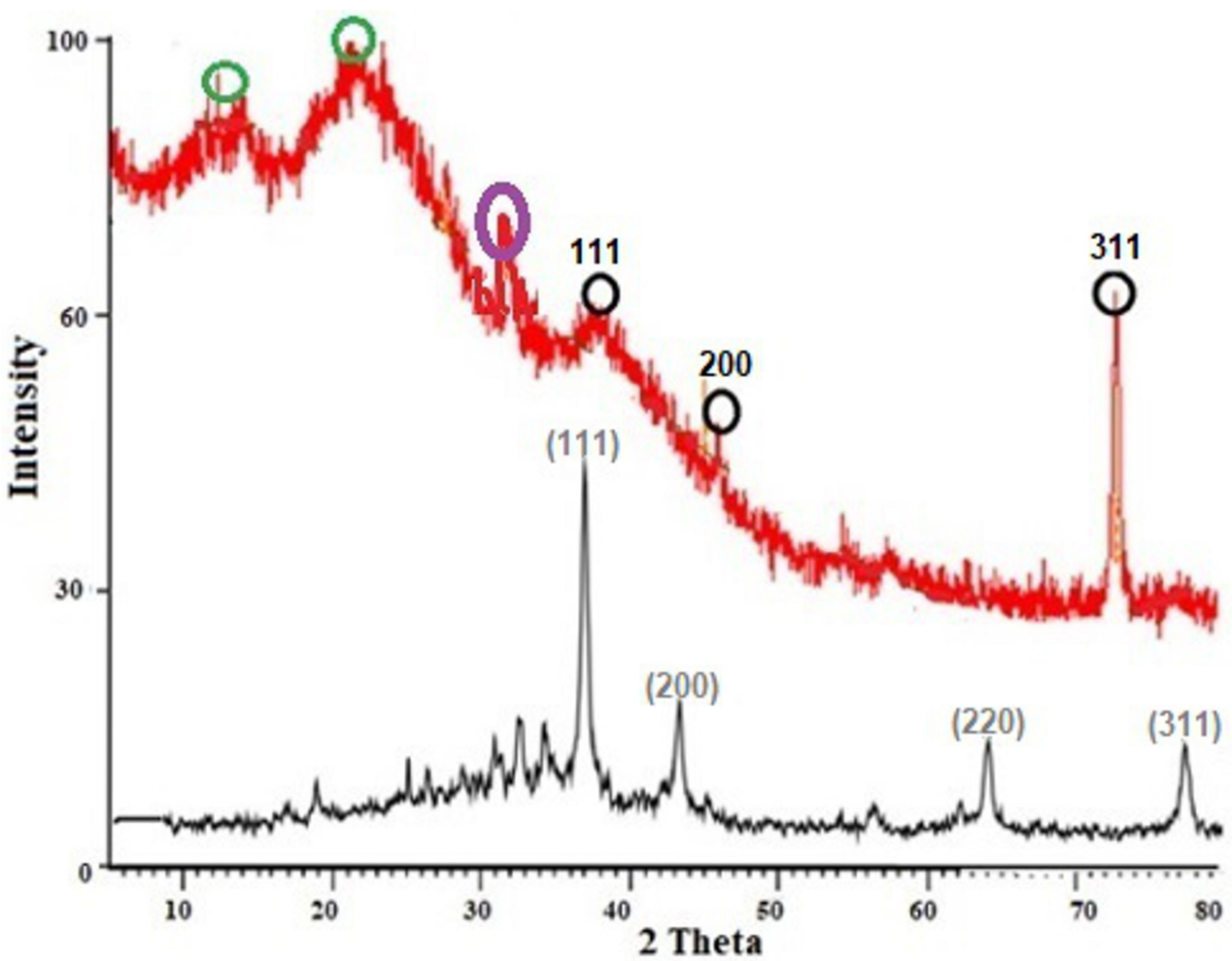

Figure 6. XRD diffraction pattern of nanocomposite hydrogel modified with AgNPs HNC.3d (red); standard AgNPs (black).

Effect of $\mathrm{AgNO}_{3}$ concentration on water swelling capacity and $\mathrm{Ag}^{+}$ion loading and release capacity

The effect of the sodium alginate to PVA ratio was studied by observing the swelling capacity in water. The results of the swelling capacity measurements are shown in Table 1. Hydrogels with higher Alg/PVA ratios had increased maximum swelling capacity. This effect is due to the increase in sodium alginate hydrophilic groups in the hydrogels rise when the $\mathrm{Alg} /$ PVA ratio is increased (Ghasemzadeh and Ghanaat, 2014).
The best swelling capacity results were observed for the Hyd. 3 formulation, which had an Alg/PVA ratio of $3: 1$ and a maximum swelling capacity of $40.72 \mathrm{~g} / \mathrm{g}$. The hydrogel was used as a nanoreactor to form silver nanoparticles using various concentrations of silver nitrate. Table 1 shows that the swelling capacity of the AgNP-modified hydrogels was higher than that of the unmodified hydrogels and that the swelling capacity increased with the concentration of silver ions. This effect is due to the presence of silver nanoparticles that form in the hydrogel matrix, 
causing an expansion of the pores and tissues in the hydrogels and thereby increasing the swelling capacity (Basu et al., 2017; Pandey et al., 2016).

The best nanocomposite hydrogel (Hyd. 3) was obtained by varying the concentration of $\mathrm{AgNO}_{3}$. Table 1 shows that higher concentrations of $\mathrm{AgNO}_{3}$ led to greater absorption of $\mathrm{Ag}^{+}$ions into the hydrogel matrix and increased $\mathrm{Ag}^{+}$ion loading, which occurred because a higher concentration of ions in the solution results in a greater frequency of collision among particles; thus, there is increased diffusion and dispersion of $\mathrm{Ag}^{+}$ions into the hydrogel matrix, resulting in increased loading of $\mathrm{Ag}^{+}$ions into the hydrogel matrix.

The silver nitrate concentration also affected the swelling capacity, as seen in Table 1. A higher initial concentration of $\mathrm{AgNO}_{3}$ resulted in a higher maximum swelling capacity because the increased $\mathrm{Ag}^{+}$ion loading inside the matrix triggers an increase in the formation of AgNPs. AgNPs inside the hydrogel matrix expand the pores, so the swelling capacity is increased.

The $\mathrm{Ag}^{+}$ion release capacity is a parameter that can be helpful in selecting the best material. The hydrogel matrix which in its swollen condition releases the absorbed silver nanoparticles because of its expanded pore structure. Thus, the hydrogel matrix confers the advantage of controlled release of silver nanoparticles and $\mathrm{Ag}^{+}$ions depending on the swelling capacity of the hydrogel material (Palza et al., 2015). Controlled release occurs because modifying nanocomposite hydrogels with AgNPs promotes swelling and the release of $\mathrm{Ag}^{+}$ions. Table 1 shows that increasing the concentration of silver nitrate leads to increased maximum swelling capacity and release of $\mathrm{Ag}^{+}$ions. Thus, a swollen hydrogel matrix can release silver ions in a controlled manner because the number of ions released depends on the amount of liquid that can be absorbed. Swelling is very useful characteristics for materials that are used as antibacterial dressings for wet wound treatments. Because the material releases only the number of needed to inhibit bacterial growth, the controlled release of $\mathrm{Ag}^{+}$ions minimize the risk to human health (Ahamed et al., 2010).

Table 1. Relationship between loading, release capacity of ion $\mathrm{Ag}^{+}$, and maximum swelling capacity in water with various initial concentrations of $\mathrm{AgNO}_{3}(\mathrm{HNC})$.

\begin{tabular}{|c|c|c|c|c|}
\hline \multirow{2}{*}{$\begin{array}{l}\text { Hydrogel } \\
\text { code }\end{array}$} & \multirow{2}{*}{$\begin{array}{c}\text { Initial } \\
\text { AgNO }_{3} \\
(\mathrm{ppm})\end{array}$} & \multirow{2}{*}{$\begin{array}{c}\text { Water Maximum } \\
\text { swelling capacity } \\
(\mathrm{g} / \mathrm{g})\end{array}$} & $\begin{array}{c}\mathbf{A g}^{+} \text {Ion } \\
\text { Loading }\end{array}$ & \multirow{2}{*}{$\begin{array}{c}\mathbf{A g}^{+} \mathbf{I o n} \\
\text { Maximum release } \\
\text { capacity }(\mathrm{ppm} / \mathrm{g})\end{array}$} \\
\hline & & & $(\mathrm{ppm} / \mathrm{g})$ & \\
\hline Hyd.1 & 0 & 24.230 & - & - \\
\hline Hyd.2 & 0 & 32.250 & - & - \\
\hline Hyd.3 & 0 & 40.720 & - & - \\
\hline HNC.3a & 6.25 & 46.463 & 6.208 & 1.110 \\
\hline HNC. $3 b$ & 31.5 & 51.433 & 25.596 & 2.583 \\
\hline HNC. $3 \mathrm{c}$ & 62.5 & 56.940 & 42.251 & 3.485 \\
\hline HNC.3d & 150 & 70.465 & 102.220 & 7.421 \\
\hline HNC.3e & 300 & 74.465 & 169.150 & 12.732 \\
\hline HNC.3f & 500 & 77.057 & 408.880 & 17.767 \\
\hline HNC. $3 \mathrm{~g}$ & 1000 & 86.591 & 676.600 & 24.082 \\
\hline
\end{tabular}

\section{In vitro antibacterial activity assay}

The in vitro antibacterial activity assay performed on the nanocomposite hydrogel was used to determine the ability of the AgNP-modified nanocomposite hydrogel to inhibit bacterial growth at various concentrations of $\mathrm{AgNO}_{3}$. The antibacterial activity of the AgNP-modified nanocomposite against $S$. aureus and $E$. coli was assessed based on the OD; the percentage inhibition and bacterial cell death were then derived using equations 5 and 6 . As shown in Table 2, the percentage inhibition increased with increasing concentrations of silver nitrate in the nanocomposite hydrogels, and a similar trend was observed for the percentage of bacterial cell death.

The percentage of bacterial cell death described the proportion of bacterial cells that can be killed in 24 hours compared to negative control and positive control. The positive control kills $100 \%$ of bacterial cells while the negative control kills $0 \%$ of bacterial cells for both types of bacteria; the percentage of bacterial death increases with increasing concentrations of silver nitrate in the hydrogel (Table 2).

Antimicrobials can inhibit the growth of bacteria at low concentrations and kill bacteria at high concentrations. The minimum inhibitory concentration (MIC) is the lowest concentration of an antimicrobial ingredient that is bacteriostatic or prevents the growth of bacteria. The minimum bactericidal concentration (MBC) is the lowest concentration of an antibacterial agent required to kill bacteria. In our work, inhibition of $E$. coli bacterial growth occurred at an $\mathrm{AgNO}_{3}$ (HNC.3c) concentration of $46.251 \mathrm{ppm}$; for $S$. aureus, $100 \%$ inhibition was achieved at $75.220 \mathrm{ppm}$ (HNC.3d). The MIC value for $S$. aureus was greater than that for $E$. coli, indicating that $S$. aureus is more resistant to $\mathrm{Ag}^{+}$ions as an antibacterial agent (Basu et al., 2017; Veiga et al., 2013). The MBC values (concentration achieving 100\% bacterial cell death) were 185.004 ppm (HNC.3f) for E. coli and 300.880 ppm (HNC.3g) for $S$. aureus (Table 2).

The MBC/MIC ratios are shown in Table 3. The $\mathrm{MBC} / \mathrm{MIC}$ ratio of the AgNP-modified nanocomposite hydrogels was four for both $E$. coli and $S$. aureus. This value reflects very good activity against both bacteria (Ghasemzadeh and Ghanaat, 2014). Thus, our results showed that the nanocomposite hydrogels have very good antibacterial activity against Grampositive and Gram-negative microorganisms, using E. coli and S. aureus as models.

Table 2. Percentage of inhibition and bacterial death.

\begin{tabular}{lccccc}
\hline \multirow{2}{*}{ HNC Code } & $\begin{array}{c}\text { Ag } \mathbf{g}^{+} \text {Ion Loading } \\
(\mathbf{p p m} / \mathbf{g})\end{array}$ & \multicolumn{2}{c}{ Inhibition (\%) } & \multicolumn{2}{c}{ Bacterial Death $(\%)$} \\
\cline { 3 - 6 } & & $\boldsymbol{E}$. coli & S. aureus & $\boldsymbol{E}$. coli & S. aureus \\
\hline+ & & 100 & 100 & 100 & 100 \\
- & 0 & 0 & 0 & 0 \\
HNC.3a & 6.208 & 40 & 10 & 18 & 9 \\
HNC.3b & 25.596 & 75 & 50 & 56 & 44 \\
HNC.3c & 46.251 & 100 & 80 & 68 & 60 \\
HNC.3d & 75.220 & 100 & 100 & 75 & 70 \\
HNC.3e & 135.150 & 100 & 100 & 85 & 80 \\
HNC.3f & 185.004 & 100 & 100 & 100 & 90 \\
HNC.3g & 300.880 & 100 & 100 & 100 & 100 \\
\hline
\end{tabular}


Table 3. MIC and MBC values of nanocomposite hydrogels modified with silver nitrate.

\begin{tabular}{lccc}
\hline Type of bacteria & MIC loading (ppm) & MBC loading (ppm) & MBC/MIC ratio \\
\hline E. coli & 46.251 & 185.004 & 4 \\
S. aureus & 75.220 & 300.880 & 4 \\
\hline
\end{tabular}

\section{CONCLUSION}

AgNP-modified nanocomposite hydrogels were successfully synthesized in this study using the post-loading method. Characterization using FTIR, SEM, TEM, XRD, and AAS showed that AgNPs were absorbed inside the hydrogel matrix. The best AgNP-modified nanocomposite hydrogels were obtained with Alg/PVA ratio of 3:1, and the silver nanoparticles in the hydrogels were $20 \mathrm{~nm}$ in size. In this study, the observed $\mathrm{AgNO}_{3} \mathrm{MIC}$ values against $E$. coli and $S$. aureus were 46.251 ppm and $75.220 \mathrm{ppm}$, respectively. The MBC values $(100 \%$ bacterial cell death) were 185.004 ppm for $E$. coli and 300.880 for $S$. aureus. Thus, the observed MBC/MIC ratio of the nanocomposite hydrogels was four for both $E$. coli and $S$. aureus. The results showed that the nanocomposite hydrogels have very good antibacterial activity against Gram-positive and Gram-negative bacteria. The developed AgNP-modified nanocomposite using sodium alginate biopolymers as the main ingredient is suitable for use in antibacterial applications in various fields, such as in dressings to promote wound healing, operating equipment, etc.

\section{FUNDING SOURCES}

This Manuscript was supported by the 2018 Publication Management Assistance Program of the University of Indonesia.

\section{CONFLICTS OF INTEREST}

The authors declare that they have no conflicts of interest.

\section{REFERENCES}

Ahamed M, AlSalhi MS, Siddiqui MKJ. Silver nanoparticle applications and human health. Clinica Chimica Acta, 2010; 411: 1841-8.

Basu S, Samanta SH, Ganguly J. Green synthesis and swelling behavior of Ag-nanocomposite semi-IPN hydrogels \& its drug delivery using Dolichos biflorus Linn. Soft Materials, 2017; 16:7-19.

Deen GS, Chua V. Synthesis and properties of new "Stimuli" responsive nanocomposite hydrogels containing silver nanoparticles. Gels, $2015 ; 1: 117-34$.

El-Sherif H, El-Masry M, Kansoh A. Hydrogels as template nanoreactors for silver nanoparticles formation and their antimicrobial activities. Macromol Res, 2011; 19(11):1157-65.

Frone A N, Nicolae C A, Gabor R A, Panaitescu D M. Thermal properties of water-resistant starch e polyvinyl alcohol films modified with cellulose nanofibers. Polym Degrad Stabil, 2015; 121(11):385-97.

Fekete T, Borsa J, Takács E, Wojnárovits L. Synthesis and characterization of superabsorbent hydrogels based on hydroxyethylcellulose and acrylic acid. Carbohyd Polym, 2017; 166:300-8.

GhavamiNejad A, Park C H, Kim C S. In situ synthesis of antimicrobial silver nanoparticles within antifouling Zwitterionic hydrogels by catecholic redox chemistry for wound healing application. Biomacromolecules, 2016; 17:1213-23.

Ghasemzadeh H, Ghanaat F. Antimicrobial alginate/PVA silver nanocomposite hydrogel, synthesis and characterization. J Polym Res, 2014: 21:355-69.
Gharekhania H, Olada A, Mirmohsenia A, Bybordi A. Superabsorbent hydrogel made of NaAlg-g-poly(AA-co-AAm) and rice husk ash: synthesis, characterization, and swelling kinetic studies. Carbohydr Polym, 2017; 168:1-13.

Helmiyati, Aprilliza M. Characterization and properties of sodium alginate from brown algae used as an ecofriendly superabsorbent. J Phys IOP Conf Ser Mater Sci Eng, 2017; 188:012019.

Helmiyati, Saefumillah A, Yulianti W. Synthesis and swelling kinetics of superabsorbent rice straw cellulose graft copolymers. Asian J Chem, 2014; 26(21):7337-42.

Helmiyati, Budianto E, Priyono W, Yulizar Y. The kinetics and mechanism of the core-shell styrene-butyl acrylate polymerisation. J Phys Sci, 2010; 21(1):39-52.

Jayakumar R, Prabaharan M, Sudheesh Kumar PT, Nair SV, Tamura H. Biomaterials based on chitin and chitosan in wound dressing applications. Biotechnol Adv, 2011; 29(3):322-37.

Lee KY, Mooney DJ. Alginate: properties and biomedical applications review article. Prog Polym Sci, 2012; 37:106-26.

Pandey S, Ramontjaa J. Sodium alginate stabilized silver nanoparticles-silica nanohybrid and their antibacterial characteristics. Int $\mathrm{J}$ Biol Macromol, 2016; 93:712-23.

Phongtongpasuk S, Poadang S, Yongvanich N. Environmental friendly method for synthesis of silver nanoparticles from dragon fruit peel extract and their antibacterial activities. Energy Procedia, 2016; 89: 239-47.

Palza H. Review antimicrobial polymers with metal nanoparticles. Int J Mol Sci, 2015; 16:2099-116.

Pereira R, Carvalho A, Vaz D C, Gil M H, Mendes A, Bártolo P. Development of novel alginate based hydrogel films for wound healing applications. Int J Biol Macromol, 2013; 52:221-30.

Raguvaran R, Manuja BK, Chopra M, Thakur R, Anand T, Kalia A, Manuja A. Sodium alginate and gum acacia hydrogels of $\mathrm{ZnO}$ nanoparticles show wound healing effect on fibroblast cells. Int J Biol Macromol, 2017; 96:185-91.

Solomon S D, Bohadory M, Aravindan V, Jeyarajasingam A V, Rutkowsky S A, Boritz C, Mulfinger L. Synthesis and study of silver nanoparticles. J Chem Educ, 2007; 84(2):322-5.

Thakur S, Sharma B, Verma A, Chaudhary J, Tamulevicius $\mathrm{S}$, Thakur V K. Recent progress in sodium alginate based sustainable hydrogels for environmental applications. J Clean Prod, 2018; 198: 143-59.

Veiga AS, Schneider JP. Review antimicrobial hydrogels for the treatment of infection. Biopolymers, 2013; 100(6):637-44.

Zhao SW, Guo CR, Hu YZ, Guo YR, Pan QJ, The preparation and antibacterial activity of cellulose/ZnO composite: a review. Open Chem, 2018; 16:9-20.

Zhao X, Xia Y, Li Q, Ma X, Quan F, Geng C, Han Z. Microwave-assisted synthesis of silver nanoparticles using sodium alginate and their antibacterial activity. Colloid Surf A, 2014; 444: $180-8$.

How to cite this article:

Helmiyati, Novientri G, Abbas GH, Budianto E. Nanocomposite hydrogel-based biopolymer modified with silver nanoparticles as an antibacterial material for wound treatment. J Appl Pharm Sci, 2019; 9(11):001-009. 